\title{
Visible Optical Resonances in Electrically Doped
}

\section{DNA}

\author{
J. R. M. Saavedra ${ }^{\dagger}$ and F. Javier García de Abajo*,†,† \\ $\dagger$ †CFO-Institut de Ciencies Fotoniques, The Barcelona Institute of Science and Technology, \\ 08860 Castelldefels (Barcelona), Spain \\ $\ddagger I C R E A$-Institució Catalana de Recerca i Estudis Avançats, Passeig Lluís Companys 23, \\ 08010 Barcelona, Spain \\ E-mail: javier.garciadeabajo@nanophotonics.es
}

\begin{abstract}
Dexoyribonucleic acid (DNA) has recently been identified as a promising material for nanotechnology due to its unique mechanical, electrical, and optical properties. However, optical applications are severely hampered by the featureless response of neutral DNA at visible frequencies. Additionally, predictive simulations are computationally too demanding to cope with large DNA strands. Here, we develop a computationally efficient procedure to simulate the optical response of large DNA molecules and reveal the emergence of electrically-tunable intense resonances in the visible spectral range. Our results support the potential of DNA for optoelectronics and biosensing applications.
\end{abstract}

Keywords: electro-optical tunability, DNA optical resonances, optical response, molecular plasmons, single-strand DNA 
Deoxyribonucleic acid (DNA) is widely known as the molecule of life, used by living organisms as a universal encoding engine. Its discovery ${ }^{1,2}$ and subsequent characterization revolutionized biology because it plays a central role in basic processes such as protein synthesis and genetic inheritance. ${ }^{3-6}$ Recently, applications of DNA have been explored beyond biology, essentially relying on its mechanical, electrical, and optical properties, which render it a promising active nanomaterial. ${ }^{7}$ In particular, its flexibility and systematic pair hybridization enable DNA origami ${ }^{8}$ and kirigami, ${ }^{9}$ which permit producing nanostructured DNA scaffolds of arbitrary morphology. ${ }^{10,11}$ Additionally, its chemical reactivity allows dynamic modification of its spatial conformation, ${ }^{12}$ which has been recently used in the so-called plasmonic walkers. ${ }^{13}$ Regarding electrical properties, the DNA double helix has been postulated as a nanoscale wiring material because it appears to be a good hole-driven conductor. ${ }^{14}$ Even in information technologies, applications of DNA have been explored as a platform for data storage ${ }^{15,16}$ and complex-problem solving. ${ }^{17-20}$

From an optical viewpoint, the response of neutral DNA presents its dipole-active electronic transitions at ultraviolet frequencies, ${ }^{21,22}$ acting as a featureless transparent dielectric in the visible spectral region below $\sim 4 \mathrm{eV} .{ }^{22} \mathrm{~A}$ similar behavior is also found in large neutral polycyclic aromatic hydrocarbons (PAHs), which however display visible plasmon-like resonances when they are electrically doped: ${ }^{23}$ the addition or removal of a single electron in these molecules can dramatically modify their optical response, introducing optical resonances in the technologically interesting visible region. ${ }^{23,24}$ Now, the question arises, does DNA display visible optical resonances when it is electrically doped? This capability, in combination with the ubiquity of DNA in biological media and the existence of techniques for manipulating its geometrical configuration, would render this genetic molecule as a promising platform for active nanophotonics.

In this article we show that DNA displays visible optical resonances when it is electrically doped, thus opening the way toward applications in light modulation and sensing. Specifically, we study the optical response of neutral and charged DNA from first principles by 
combining time-dependent density-functional theory (TDDFT) to describe individual nucleobases $(\mathrm{NBs})$ and classical inter-NB interaction to deal with large DNA molecules. We further introduce an efficient scheme to express the optical response in terms of polaritonic wave functions (PWFs), which we define in order to capture quantum-mechanical atomistic details of the NB responses, while allowing us to cope with single strands composed of many NBs. We present a detailed study of individual NBs and large single DNA strands that reveals the strong electro-optical response of this material.

\section{RESULTS AND DISCUSSION}

Despite its importance in the understanding of biologically relevant processes, the realistic simulation of the optical response of large DNA strands constitutes a numerically demanding problem that becomes rapidly impractical when the number of NBs increases. ${ }^{25}$ As a way to tackle this problem, we use in our study a radical approximation, consisting in assuming that the excitations in each NB involve only electronic orbitals that do not overlap with either the deoxyribose backbone or the immediate NB neighbors. The first of these approximations should be safe because the backbone features resonances only at high excitation energies above $\sim 7 \mathrm{eV} .{ }^{26}$ Regarding interaction between nearest NB neighbors, their relatively large separation (see Figure 1a) along a single DNA strands prevents direct overlap of their electronic charge. Under this approximation, we can describe the response of each NB from first principles and account for inter-NB coupling through the Coulomb interaction between the resulting induced charges. In practice, we obtain the response of each NB from first principles and express it in terms of PWFs defined by the corresponding induced charge density distributions (see Methods). The remaining Coulomb interaction among different NBs in the DNA chain is then described expressed as matrix elements involving their PWFs. 
a)

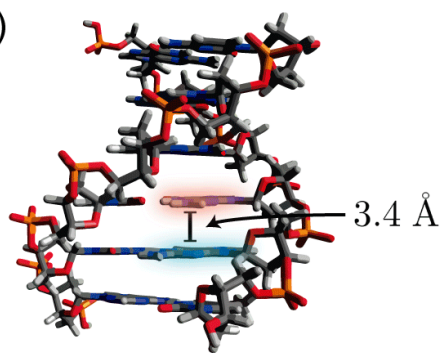
PWF: - Positive - Negative - Neutral
b) c)
TDDFT: $\cdots$ Positive $\cdots$ Negative $\cdots$ Neutral

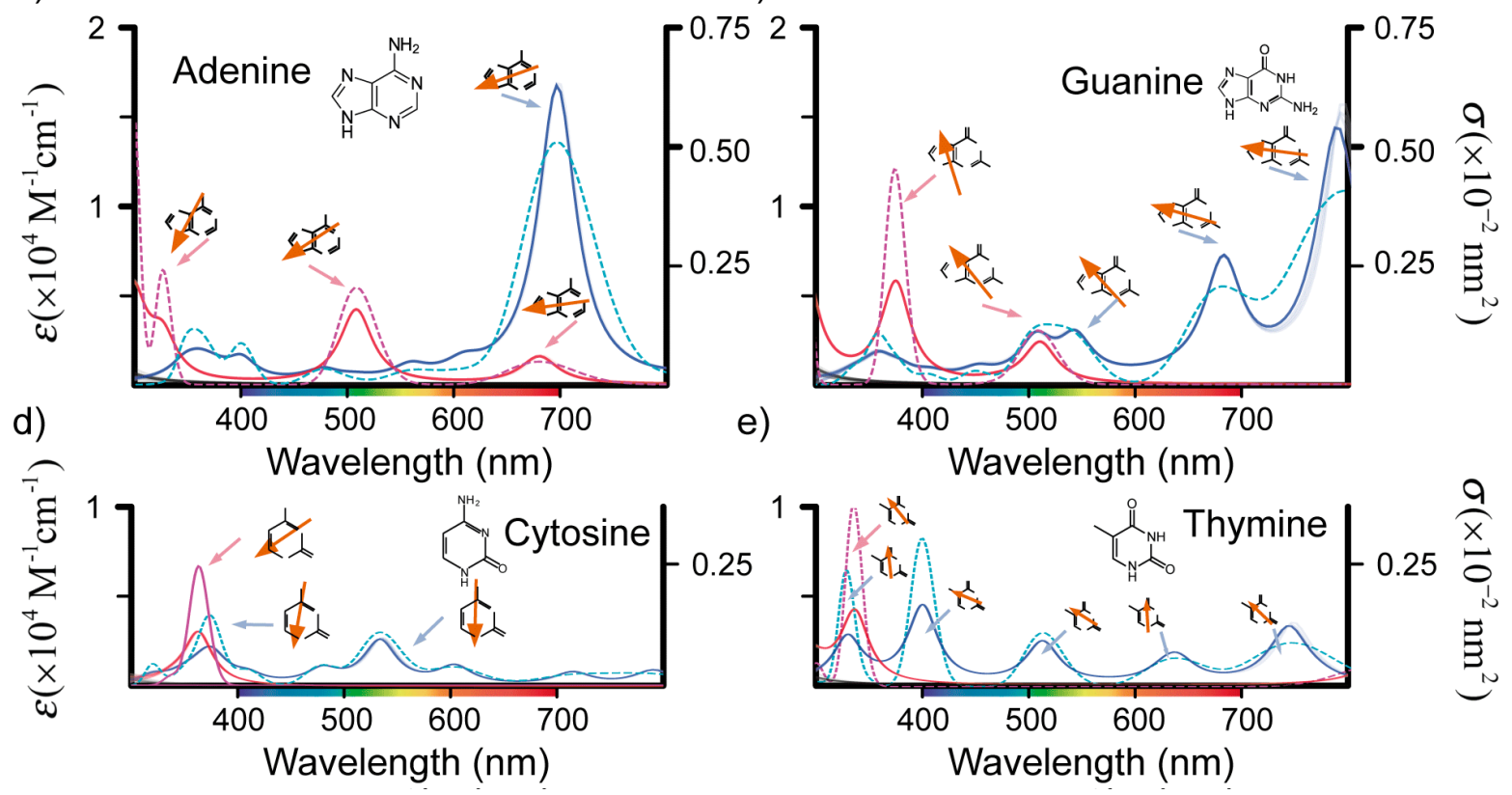

Figure 1: Optical response of charged DNA nucleobases (NBs) in the visible regime. a Atomic structure of a double-strand DNA showing a characteristic example of the position and distance of neighboring NBs. b-e We show the molar attenuation coefficient (left vertical axes) and absorption cross-section (right axes) for each of the four possible types of NBs with positive (red) or negative (blue) doping (i.e., by removing or adding one electron, respectively). The spectra for neutral NBs (gray) takes much smaller values and is only visible in the lower-left part of the plots. Simulations for individual NBs performed by TDDFT (dashed curves) are compared with polaritonic wave function (PWF) calculations (solid curves) in which each charged NB X is flanked by two other NBs in a single-strand DNA (sDNA) YXZ configuration. We plot different realizations of the latter (i.e., YXZ, with $\mathrm{X}$, Y, and $\mathrm{Z}$ running over the four types of NBs), which are hardly distinguishable on the scale of the plots. The orientations of the induced dipoles associated with different spectral features are shown as insets. 


\section{Optical response of individual charged nucleobases}

We first discuss the optical properties of neutral and charged individual NBs as a preliminary step in our study. Figure 1b-e presents the results for the optical response of all four DNA NBs obtained from TDDFT Gaussian simulations ${ }^{27}$ (see details in Methods and Supplementary Information, SI). We compare different charge states of the molecules: neutral (gray curves) and doped with an additional electron (blue) or hole (red). We concentrate on the technologically relevant visible range, in which we observe that neutral NBs present a weak featureless absorption, in agreement with results in the literature ${ }^{26}$ (i.e., their electronic optical resonances are concentrated in the ultraviolet, with minor overlap with the visible region). In contrast, the four NBs exhibit strong visible resonance features under positive or negative doping. Additionally, they exhibit major differences in their absorption spectra between different bases, but also when comparing and positive and negative doping in the same base.

The peak molar attenuation coefficients are of the order of $10^{4} \mathrm{M}^{-1} \mathrm{~cm}^{-1}$, with purines (adenine (A) and guanine $(\mathrm{G})$ ) displaying roughly a factor of 2 higher peaks than pyrimidines (cytosine $(\mathrm{C})$ and thymine $(\mathrm{T})$ ). These numbers have the same order of magnitude as the ultraviolet resonances of the neutral molecules. Incidentally, in our study the peak absorption is directly proportional to the resonance lifetime, for which we use a plausible frequency-dependent empirical estimate (see Methods). Nevertheless, the peak area is roughly independent of this parameter. The strongest resonances are observed in negatively charged adenine at around $700 \mathrm{~nm}$, while the response spreads over the visible spectrum and is peaked at $500 \mathrm{~nm}$ when the molecule is positively charged. Similarly drastic variations with the sign of the doping are observed in the other three molecules

In addition to the absorption spectra, we show the orientation of the transition dipoles for each of the relevant resonances in the spectra. Many of these dipoles are directed along the symmetry axis of the molecule (see insets), with a slight deviation toward regions of higher electronegativity. 
The TDDFT simulations presented in Figure 1 are strictly valid only if the NBs are isolated. As discussed above, we intend to study the interaction among NBs in DNA strands using the PWF formalism under the assumption of negligible mutual electronic overlap. In order to corroborate the validity of this approach, we also show in Figure 1b-e calculations based on this PWF formalism for sequences of three NBs, with the central one in either neutral or doped states. This analysis reveals a marginal influence of neutral neighboring NBs on the central NB is small (i.e., all combinations of neutral neutral neighbors produce nearly indistinguishable spectra for each choice of central NB). Additionally, the results are similar to those obtained from TDDFT simulations, except for a variation in linewidth between Gaussian (constant width of $0.1 \mathrm{eV}$ across the spectrum) and PWF simulations (frequencydependent width, see Methods). This further confirms a low degree of hybridization between the charge densities of the excited states in the NBs. Although this does not affect our study, we observe major effects associated with hybridization in the UV regime (not shown), where the different excited states of the surrounding neutral NBs affect the optical response of the entire NB chain.

\section{Interaction between neighboring charged NBs}

The above results suggest that the optical response of charged NBs is largely unaffected by the presence of neutral NBs. However, neighboring doped NBs can exert a mutual influence on each other because both of them contain modes in the visible region, thus facilitating hybridization through their Coulomb interaction. This type of interaction can other when electrically doping single-strand DNA (sDNA) with a large density of charges (see below), but it also occurs naturally in exciplexes, which are defects formed by charge transfer between neighboring NBs and arise naturally in the relaxation process associated with UV excitation of neutral DNA. ${ }^{22}$ We analyze these configurations under the assumption that the charges (positive and/or negative) are entirely localized within each of the neighboring NBs. ${ }^{28}$ This assumption allows us to apply the PWF formalism in order to estimate their effect on the 
optical response of DNA.
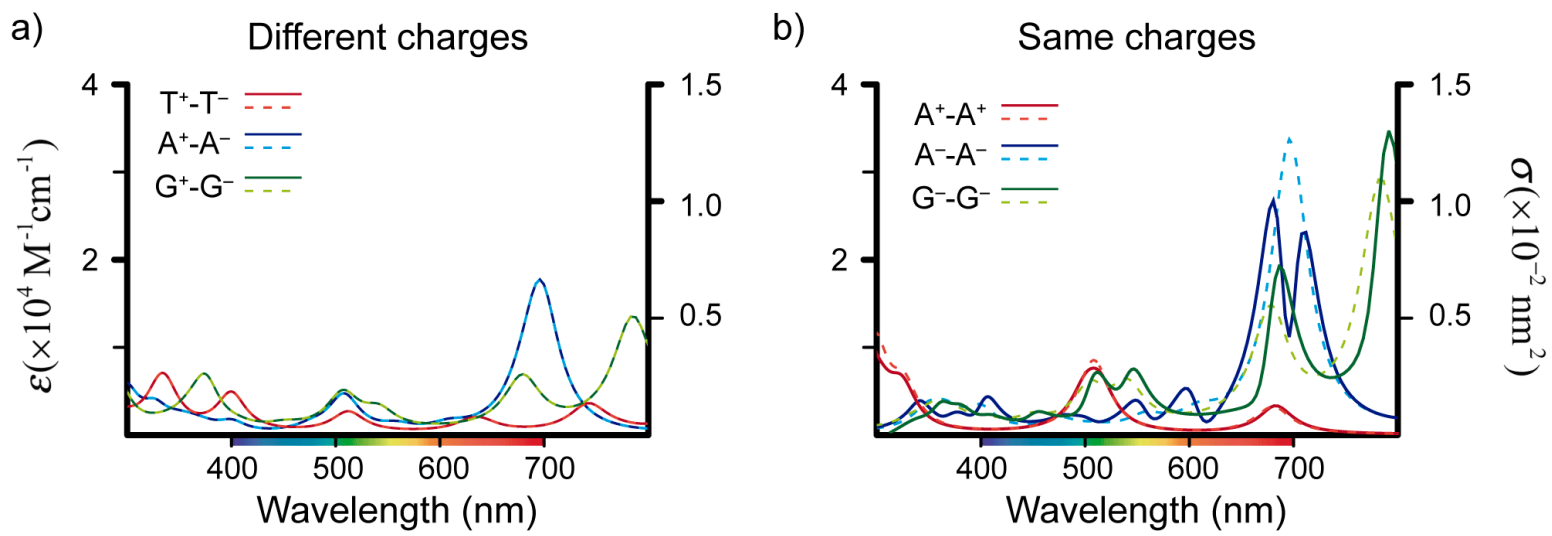

Figure 2: Visible optical response of neighboring charged NBs. a Absorption spectra of exciplexes formed by different combinations of NBs with opposite charges. b Absorption spectra produced by identical neighboring charged NBs. Solid (dashed) curves are PWF calculations with (without) inclusion of Coulomb coupling between the two neighboring NBs.

We start by analyzing the optical response of exciplexes in Figure 2a, where we consider three different examples of neighboring NBs, but the results are qualitatively similar in all kinds of possible combinations. A first conclusion from these calculations is that the exciplexes display a strong optical response in the visible region, in contrast to the neutral bases, which show essentially negligible absorption. Additionally, the response is given to a good approximation as the sum of the responses of the charged NBs that form the exciplex (cf. solid and dashed curves in Figure 2a). We interpret this lack of interaction between neighboring NBs as the result of the mismatch in their absorption resonances (see Figure 1). Exciplexes are thus a rather simple example of charged sDNA that can be formed by simply irradiating with UV light, and whose optical response is easy to interpret as the direct sum of their charged constituents.

This situation changes dramatically when considering symmetric pairs of charged NBs (see Figure 2b): the interaction between resonances at the same frequency in each of the neighboring NBs can lead to strong modifications in the optical response (cf. solid and dashed 
curves in Figure 2b). This effect is particularly strong in $\mathrm{A}^{-}-\mathrm{A}^{-}$, which was expected from the results of Figure 1 because $\mathrm{A}^{-}$shows the strongest resonances among the charged NBs.

\section{Optical response of multiply-charged sDNA}

Potential applications of charged DNA for optoelectronics could involve the presence of multiple carriers along the DNA. In practice, one end of a sDNA chain could be attached to a conductive surface (e.g., an optically featureless transparent electrode) and be subject to a strong DC electric field $\mathbf{E}_{\mathrm{DC}}$ produced by another neighboring (but not in contact) electrode. Noticing that DNA is an excellent conductor mediated by holes ${ }^{29}$ (i.e., they can migrate along several bases without being reabsorbed), we consider the presence of multiple positive charges as a likely situation, although we also analyze doping with multiple negative charges, which could be achieved with a suitable choice of gates. Despite the fact that our approach does not attempt to control charging of specified NBs along the chain, it provides a guidance on how the interaction among multiple charges may be used to further tailor the optical response of a doped DNA chain. We then need to address two problems: the self-consistent charge distribution along the chain; and the resulting self-consistent optical response. We solve the first of them by minimizing the total energy of the system (Coulomb repulsion plus NB ionization energies, see Methods), while the second one is computed using the PWF formalism.

In Figure 3a, we study a sDNA chain consisting of 50 NBs with a NB-type distribution similar to human DNA (see Methods). We assume that the molecule is attached to a gate that can introduce charges along the chain, and further consider the presence of a gating DC field $E_{\mathrm{DC}}=0.3 \mathrm{~V} / \mathrm{nm}$ (Figure $3 \mathrm{~b}$ ). In practice, different DNA terminations can be contemplated that produce binding to the gate, although for simplicity we dismiss the effect of both the gate material and the binding molecular termination on the resulting optical response. We use the PWF formalism in our simulations, taking into account the helical structure of DNA, but ignoring further secondary structure for simplicity. As expected, we 

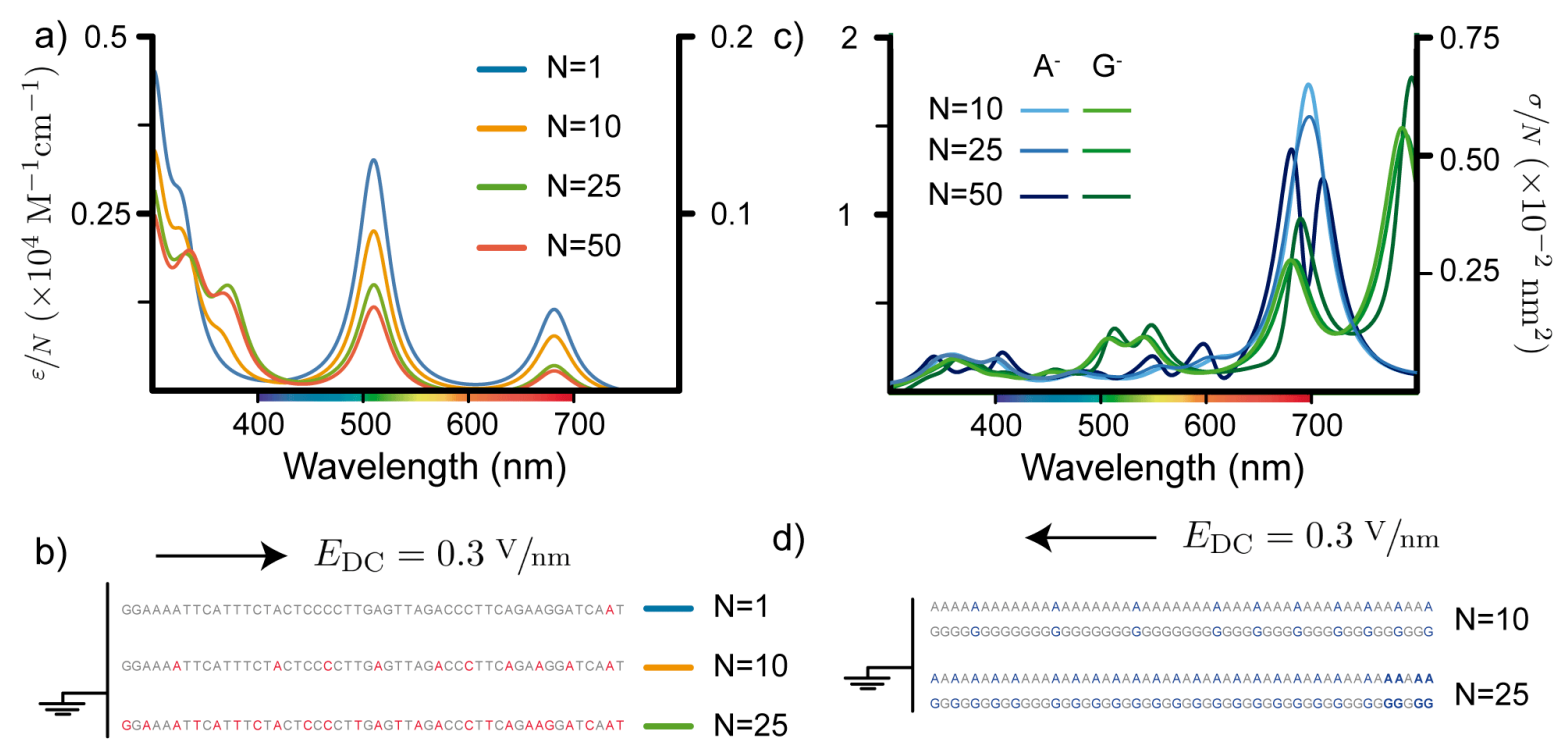

d)

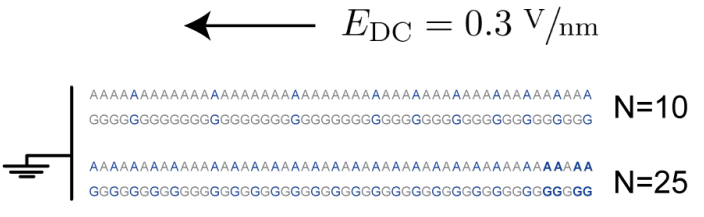

Figure 3: Variation of the optical response with the number of charges present in a sDNA chain. We study the variation in the absorption cross-section of a positively charged sDNA chain as we increase the number $N$ of positively charged NBs in the molecule. a,b We show in a the absorption spectra corresponding to different values of $N$ (normalized to $N$ in each case) with a distribution of positive charges as shown in b. We consider a 50NB chain, randomly generated with the same NB abundance as in human DNA, and with a self-consistent distribution of the positive charges that takes into account their electrostatic interactions, the NB ionization energies, and the presence of a bias DC field of $0.3 \mathrm{~V} / \mathrm{nm}$ (see Methods). c,d Same as a,b for two different homogeneous sDNA chains composed of either A or G and negative doping charges. Charges injected in a,b and c,d are positive and negative, respectively, as determined by where the sDNA chain is attached to a lead. 
find a strong visible response produced by the presence of doping charges, which builds up as their number increases. Additionally, the spectral profiles change with the number of added charges because of the involvement of a different mix of NBs. Nevertheless, similar to Figure 2a, the response is essentially composed of the addition of the individual response of NBs, with minor effects associated with inter-NB interactions. We obtain qualitatively similar conclusions when considering homogeneous sDNA chains (Figure 3c,d), except that the spectral response also changes here as the number of doping charges increases because of the enhanced nearest-neighbor interactions when they are identical (see also Figure 2b).

\section{Charged double-strand DNA}

DNA is commonly arranged in a double helix through Watson-Crick pair formation. When this occurs, hydrogen bonds in adenine-thymine (A-T) and cytosine-guanine (C-G) NB pairs prevents the use of PWFs with a basis set restricted to single NBs. We note that an extension of this method can still be applied when PWFs are defined for the A-T and C-G combinations. We present results for charged Watson-Crick pairs in Figure 4a, where we observe radical changes in the optical response with respect to neutral pairs. However, only a small part of these changes emerge in the visible region, essentially as very broad absorption features whose magnitude is significantly weaker than in sDNA. This effect seems to be associated with the redistribution of the added charge among the two NBs in the pair, so that each of them is doped with a fraction of an elementary charge (in contrast to doping in sDNA) and therefore undergoes a comparatively smaller modification in its optical response.

\section{CONCLUDING REMARKS}

In summary, we have studied the optical response of charged DNA nucleobases, showing that the addition or subtraction of an electron induces radical changes in their optical responses, including the emergence of resonances in the visible spectrum, where the molecules show 


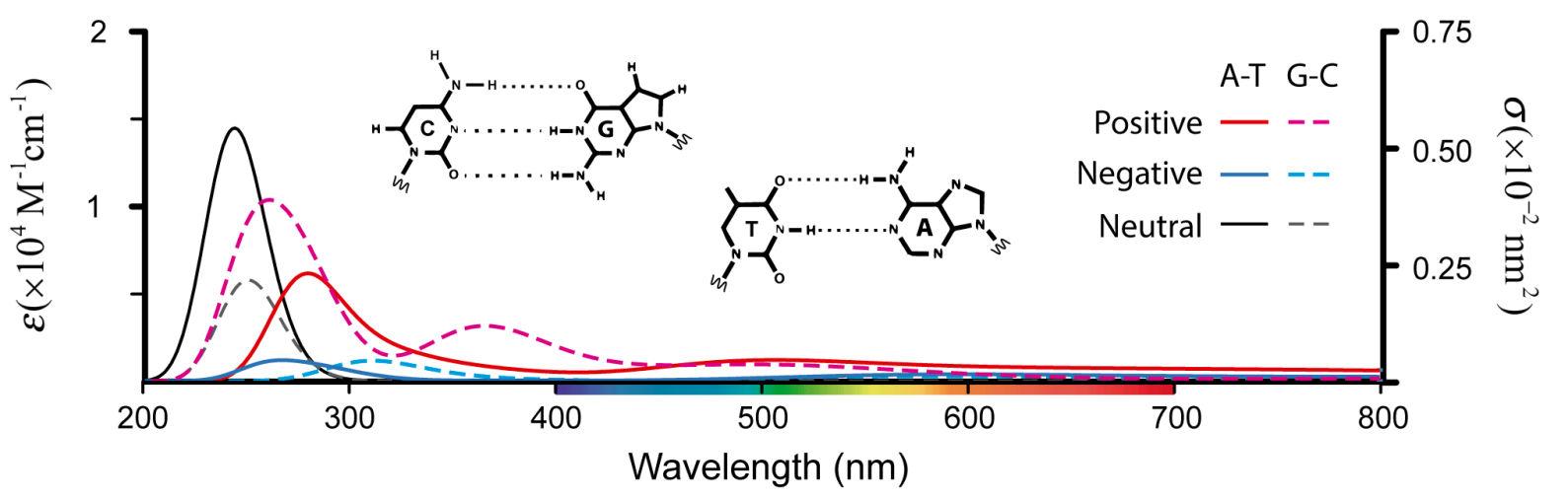

Figure 4: Effect of charging in double-strand DNA (dDNA). Absorption spectra of charged Watson-Crick pairs.

a featureless response in the absence of charging. These results can find a wide range of applications in view of the ubiquity of DNA, combined with the availabilty of methods to manipulate and integrate it in nanostructured environments. The potential of DNA for optoelectronics is supported by our results because this molecule is conducting, so under the exposure to the strong DC fields produced in gating devices, it could undergo color changes, thus molding the spectral transmission on demand. As a main conclusion of our work, we show that sDNA displays a much stronger response than dDNA in the visible, thefore making it more suitable for applications. Although a common way of doping nanostructures (e.g., graphene ${ }^{30}$ ) consists in physically attaching them to gates that provide charge injection, we anticipate the strong potential of sDNA to display an electrically tunable visible response by exposing large chains to DC fields, whereby the molecules remain neutral, although charges of opposite sign are induced in them to minimize their energy in those fields. Alternatively, one could also bridge gates with DNA molecules, through which charges can hop between nucleobases, therefore producing transient charged states that change their visible optical responses as predicted in this work. 


\section{METHODS}

TDDFT simulations. We perform first principle simulations of the optical response of individual NBs in isolation using the Gaussian 16 computational chemistry suite. ${ }^{27}$ We first carry out a DFT optimization of the atomic structure, followed by the computation of the first 20 excited states by means of TDDFT. All calculations are performed at the B3LYP/6$311++\mathrm{G}$ level of theory. The basis set $6-311++\mathrm{G}$ is a compromise between physical accuracy and computational efficiency, as we show in the SI.

PWF formalism. Inspired by a previously formulated classical description of graphene plasmonic nanostructures, ${ }^{31,32}$ we define the PWF associated with each optical resonance $j$ of the NBs as the charge-density distribution $\rho_{j}(\mathbf{r})=-e\left\langle e_{j}|\hat{\rho}(\mathbf{r})| g\right\rangle$, where $|g\rangle$ is the manybody ground state of the molecule and $\left|e_{j}\right\rangle$ is the corresponding excited state. In the TDDFT formalism, the states are linear combinations of Slater determinants $|S\rangle$ (extracted from Gaussian), so we first calculate $\left\langle S|\hat{\rho}(\mathbf{r})| S^{\prime}\right\rangle$ for all combinations $S, S^{\prime}$ of such determinants, from which we readily obtain $\rho_{j}(\mathbf{r})$.

For an individual NB, we use the well-established formalism of linear-response theory. ${ }^{33}$ We neglect retardation due to the small size of the molecule, which allows us to express the optical electric field in terms of the scalar potential $(\mathbf{E}=-\nabla \phi)$, and further consider monochromatic fields of frequency $\omega$ (i.e., $\left.\phi(\mathbf{r}, t)=2 \operatorname{Re}\left\{\phi(\mathbf{r}) \mathrm{e}^{-\mathrm{i} \omega t}\right\}\right)$. The charge density induced on the molecule in response to an externally applied potential (e.g., $\phi^{\text {ext }}(\mathbf{r})=-\mathbf{E}_{0} \cdot \mathbf{r}$ for incident light of electric field $\mathbf{E}_{0}$ at the molecule) can be written as

$$
\rho^{\text {ind }}(\mathbf{r})=\int d^{3} \mathbf{r}^{\prime} \chi\left(\mathbf{r}, \mathbf{r}^{\prime}, \omega\right) \phi^{\operatorname{ext}}\left(\mathbf{r}^{\prime}, \omega\right)
$$

where the susceptibility

$$
\chi\left(\mathbf{r}, \mathbf{r}^{\prime}, \omega\right)=\sum_{j} D_{j}(\omega) \rho_{j}(\mathbf{r}) \rho_{j}\left(\mathbf{r}^{\prime}\right)
$$


is readily expressed in terms of the molecular PWFs, the sum runs over excitations $j$ (frequency $\omega_{j}$ and width $\gamma_{j}$ ), and the coefficients

$$
D_{j}(\omega)=\frac{e^{2}}{\hbar} \frac{2 \omega_{j}}{(\omega+\mathrm{i} \gamma)^{2}-\omega_{j}^{2}}
$$

capture the dependence on $\omega$. From these expressions, we can readily write the charge density $\rho^{\text {ind }}(\mathbf{r})=\sum c_{j} \rho_{j}(\mathbf{r})$ as a combination of PWFs.

We now express the response of a sDNA chain in terms of the susceptibilities of its NBs, centered at positions $\mathbf{r}_{l}$ and labeled by $l$. Assuming no electronic overlap between neighboring NBs, the charge density is the sum of contributions from different molecules, so it can be rewritten as

$$
\rho^{\mathrm{ind}}(\mathbf{r})=\sum c_{l j} \rho_{l j}\left[\mathbf{U}_{l} \cdot\left(\mathbf{r}-\mathbf{r}_{l}\right)\right]
$$

where the coefficients $c_{l j}$ and the PWFs $\rho_{l j}$ now depend on the NB label $l$. Here, $\mathbf{U}_{l}$ are base-dependent rotation matrices that compensate for the orientation of the NB in the chain in the spatial coordinate on which the PWFs depends. This allows us to calculated the PWFs once and for all for each of the four types of NBs and apply them to arbitrary NB orientations. We now obtain the coefficients $c_{l j}$ by applying eq 1 to each NB, with $\phi^{\text {ext }}$ replaced by the light potential plus the potential created by the rest of NBs in the chain. After a tedious but straightforward calculation, this leads to the self-consistent relation

$$
c_{l j}=D_{l j}(\omega)\left[c_{l j}^{0}+\sum_{l^{\prime} \neq l} \sum_{j^{\prime}} M_{l l^{\prime} j^{\prime}} c_{l^{\prime} j^{\prime}}\right],
$$

where

$$
c_{l j}^{0}=\int d^{3} \mathbf{r} \rho_{l j}\left[\mathbf{U}_{l} \cdot\left(\mathbf{r}-\mathbf{r}_{l}\right)\right] \phi^{\operatorname{ext}}(\mathbf{r})
$$


gives the direct interaction with the external potential, while the coefficients

$$
M_{l j l^{\prime} j^{\prime}}=\iint d \mathbf{r} d \mathbf{r}^{\prime} \frac{\rho_{l j}\left(\mathbf{U}_{l} \cdot \mathbf{r}\right) \rho_{l^{\prime} j^{\prime}}\left(\mathbf{U}_{l^{\prime}} \cdot \mathbf{r}^{\prime}\right)}{\left|\mathbf{r}_{l}-\mathbf{r}_{l^{\prime}}+\mathbf{r}-\mathbf{r}^{\prime}\right|}
$$

describe the Coulomb interaction among PWFs in different NBs. Incidentally, in the limit of large separations $\mathbf{R}_{l l^{\prime}}=\mathbf{r}_{l}-\mathbf{r}_{l^{\prime}}$ compared with the size of the PWF distributions, we can Taylor-expand the Coulomb interaction in eq 2 around $\mathbf{r}-\mathbf{r}^{\prime}=0$ and use the condition of constant net charge within each NB to approximate $M_{l j l^{\prime} j^{\prime}} \approx \mathbf{p}_{l j} \cdot \mathbf{p}_{l^{\prime} j^{\prime}} / R_{l l^{\prime}}^{3}-3\left(\mathbf{R}_{l l^{\prime}} \cdot \mathbf{p}_{l j}\right)\left(\mathbf{R}_{l l^{\prime}}\right.$. $\left.\mathbf{p}_{l^{\prime} j^{\prime}}\right) / R_{l l^{\prime}}^{5}$, which thus reduces to the electrostatic dipole-dipole interaction.

Throughout this paper we plot extinction cross-sections $\sigma=(4 \pi \omega / c) \operatorname{Im}\left\{\mathbf{p} \cdot \mathbf{E}_{0}^{*}\right\} /\left|E_{0}\right|^{2}$,

here expressed in terms of the total induced dipole $\mathbf{p}=\int d^{3} \mathbf{r} \mathbf{r} \rho^{\text {ind }}(\mathbf{r})$ by virtue of optical theorem. ${ }^{34}$ Finally, taking again into account that the total charge in each NB remains constant, we can write $\mathbf{p}=\sum_{l j} c_{l j} \mathbf{p}_{l j}$ in terms of the dipoles

$$
\mathbf{p}_{l j}=\int d^{3} \mathbf{r} \mathbf{r} \rho_{l j}\left(\mathbf{U}_{l} \cdot \mathbf{r}\right)
$$

associated with the PWFs.

Self-consistent charge arrangement in multiply-charged sDNA chains. We assume that each charge carrier in the structure is entirely placed in a single NB. The positions of the charges when multiple carriers are introduced is then determined by minimizing the energy change in the system

$$
E_{\mathrm{tot}}=\sum_{l=1} \Delta E_{l}^{q_{l}}+\frac{1}{2} \sum_{l, l^{\prime}} \frac{q_{l} q_{l^{\prime}}}{\left|\mathbf{r}_{l}-\mathbf{r}_{l^{\prime}}\right|}-\sum_{l=1} q_{l} \mathbf{E}_{\mathrm{DC}} \cdot \mathbf{r}_{l}
$$

where $q_{l}(=-1,0$, or 1$)$ is the excess of charge in the NB $l$ and $\Delta E_{l}^{q_{l}}$ is the total change of the NB $l$ relative to its neutral state. For simplicity, we have approximated the charge in each $\mathrm{NB}$ as point at their respective centroid positions $\mathbf{r}_{l}$. The rightmost terms in this expression is the energy associated with a bias DC electric field $\mathbf{E}_{\mathrm{DC}}$. Using DFT from Gaussian (see 
above), we find $\Delta E_{l}^{+}=4.4 / 4.6 / 4.6 / 5.4 \mathrm{eV}$ and $\Delta E_{l}^{-}=-4.1 /-4.1 /-3.8 /-4.4 \mathrm{eV}$ for $\mathrm{A} / \mathrm{C} / \mathrm{G} / \mathrm{T}$ NBs.

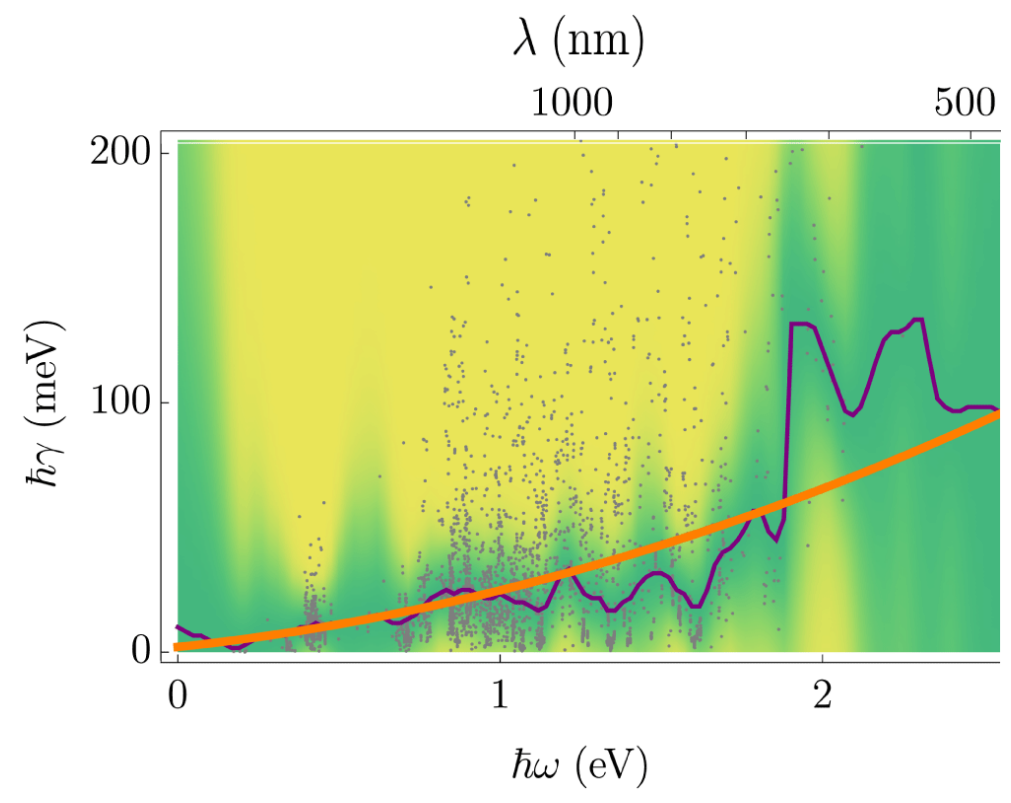

Figure 5: Variation of the resonance width with photon energy in molecular excitations. The dots in the plot correspond to molecular excitations (fitted to Lorentzian profiles) for the large set of modes and molecules tabulated in the HITRAN database. ${ }^{35}$ The color density plot (yellow is low and green is high) is obtained through a Lorentzian broadening of the dots. We also show the average width as a function excitation energy (purple curve) and a quadratic fit to this function (orange curve, see main text).

Optical resonance widths. The optical resonances under considerations have a lifetime mainly limited by coupling to atomic vibrations in the DNA and its environment. We introduce this effect through a phenomenological decay rate $\gamma$. This parameter, which can depend on the specific molecular configuration, is not available for optical resonances in charged DNA. As a reasonable estimate, we approximate it to average values obtained upon inspection of a set of experimentally measured decay rates for a large collection of organic molecules (the HITRAN database ${ }^{35}$ ). The corresponding analysis is presented in Figure 5, from which we obtain a mode-frequency-dependent broadening $\hbar \gamma / \mathrm{meV}=$ $2.07+13.7(\hbar \omega / \mathrm{eV})+9.01(\hbar \omega / \mathrm{eV})^{2}$ (Figure 5, orange curve), which we use in our simulations of the DNA response. 
Table 1: $P\left(x_{i} \mid x_{i-1}\right)$ and $P\left(x_{1}\right)$ for human DNA.

\begin{tabular}{c|cccc|c}
$x_{i-1}$ & $P\left(\mathrm{~A} \mid x_{i-1}\right)$ & $P\left(\mathrm{C} \mid x_{i-1}\right)$ & $P\left(\mathrm{G} \mid x_{i-1}\right)$ & $T\left(\mathrm{~A} \mid x_{i-1}\right)$ & $P\left(x_{1}\right)$ \\
\hline $\mathrm{A}$ & 0.423 & 0.151 & 0.168 & 0.258 & 0.345 \\
$\mathrm{C}$ & 0.399 & 0.184 & 0.063 & 0.354 & 0.158 \\
$\mathrm{G}$ & 0.314 & 0.189 & 0.176 & 0.321 & 0.159 \\
$\mathrm{~T}$ & 0.258 & 0.138 & 0.187 & 0.415 & 0.337
\end{tabular}

Generation of random human-DNA sequences. We have generated the strand presented in Figure 3 using a Markov chain intended to render realistic human DNA configurations. Specifically, the first element of the strand $x_{1}$ obeys an aggregated probability distribution $P\left(x_{1}\right)$, while each of the subsequent elements $x_{i}>1$ is generated from the conditional probabilities $P\left(x_{i} \mid x_{i-1}\right)$ for the appearance of base $x_{i}$ after $x_{i-1}$. We show the values used for $P\left(x_{1}\right)$ and $P\left(x_{i} \mid x_{i-1}\right)$ in Table 1.

\section{Supporting Information}

The Supporting Information is available free of charge on the ACS Publications website at DOI: 10.1021/acsnano.xxx, where we show a comparison of spectra obtained for charged adenine using Gaussian 16 with basis sets 6-31G, 6-311++G, and 6-311+G(d,p), as well as estimates of computation times for 1-3 NBs using this Quantum Chemistry package compared with our PWF formalism.

\section{Acknowledgments}

This work has been supported in part by the Spanish MINECO (MAT2017-88492-R, SEV20150522, and PCIN-2015-155), the ERC (Advanced Grant 789104-eNANO), the Catalan CERCA Program, Fundació Privada Cellex, and AGAUR (2017 SGR 1651 and FI_B 00492-2015). 


\section{References}

1. Watson, J. D.; Crick, F. H. C. The Structure of DNA. Cold Spring Harbor Symposia on Quantitative Biology. 1953; pp 123-131.

2. Klug, A. Rosalind Franklin and the Double Helix. Nature 1974, 248, 787-788.

3. Darnell, J. E.; Lodish, H.; Baltimore, D. Molecular Cell Biology; Scientific American Books: New York, 1990; Vol. 2.

4. Shendure, J.; Ji, H. Next-Generation DNA Sequencing. Nat. Biotech. 2008, 26, 11351145.

5. Pierce, B. A. Genetics: A Conceptual Approach; Macmillan: New York, 2012.

6. Gall, J. G. DNA Replication and Beyond. Nat. Rev. Mol. Cell Biol. 2016, 17, 464.

7. Seeman, N. C.; Sleiman, H. F. DNA Nanotechnology. Nat. Rev. Mater. 2017, 3, 17068.

8. Rothemund, P. W. Folding DNA to Create Nanoscale Shapes and Patterns. Nature 2006, 440, 297-302.

9. Han, D.; Pal, S.; Liu, Y.; Yan, H. Folding and Cutting DNA into Reconfigurable Topological Nanostructures. Nat. Nanotech. 2010, 5, 712-717.

10. Pinheiro, A. V.; Han, D.; Shih, W. M.; Yan, H. Challenges and Opportunities for Structural DNA Nanotechnology. Nat. Nanotech. 2011, 6, 763-772.

11. Zhang, F.; Nangreave, J.; Liu, Y.; Yan, H. Structural DNA Nanotechnology: State of the Art and Future Perspective. J. Am. Chem. Soc. 2014, 136, 11198-11211.

12. Zhang, D. Y.; Seelig, G. Dynamic DNA Nanotechnology Using Strand-Displacement Reactions. Nat. Chem. 2011, 3, 103-113. 
13. Zhou, C.; Duan, X.; Liu, N. A Plasmonic Nanorod that Walks on DNA Origami. Nat. Commun. 2015, 6, 8102.

14. Endres, R. G.; Cox, D. L.; Singh, R. R. P. Colloquium: The Quest for High-Conductance DNA. Rev. Mod. Phys. 2004, 76, 195-214.

15. Church, G. M.; Gao, Y.; Kosuri, S. Next-Generation Digital Information Storage in DNA. Science 2012, 337, 1628.

16. Goldman, N.; Bertone, P.; Chen, S.; Dessimoz, C.; LeProust, E. M.; Sipos, B.; Birney, E. Towards Practical, High-Capacity, Low-Maintenance Information Storage in Synthesized DNA. Nature 2013, 494, 77-80.

17. Adleman, L. M. Molecular Computation of Solutions to Combinatorial Problems. Science 1994, 266, 1021-1024.

18. Qian, L.; Winfree, E. Scaling Up Digital Circuit Computation with DNA Strand Displacement Cascades. Science 2011, 332, 1196-1201.

19. L. Qian, L.; Winfree, E.; Bruck, J. Neural Network Computation with DNA Strand Displacement Cascades. Nature 2011, 475, 368-372.

20. Currin, A.; Korovin, K.; Ababi, M.; Roper, K.; Kell, D. B.; Day, P. J.; King, R. D. Computing Exponentially Faster: Implementing a Non-Deterministic Universal Turing Machine Using DNA. J. R. Soc. Interface 2017, 14, 20160990.

21. Tsolakidis, A.; Kaxiras, E. A TDDFT Study of the Optical Response of DNA Bases, Base Pairs, and Their Tautomers in the Gas Phase. J. Phys. Chem. A 2005, 109, 2373-2380.

22. Middleton, C. T.; de La Harpe, K.; Su, C.; Law, Y. K.; Crespo-Hernńadez, C. E.; Kohler, B. DNA Excited-State Dynamics: From Single Bases to the Double Helix. Ann. Rev. Phys. Chem. 2009, 60, 217-239. 
23. Manjavacas, A.; Marchesin, F.; Thongrattanasiri, S.; Koval, P.; Nordlander, P.; SánchezPortal, D.; García de Abajo, F. J. Tunable Molecular Plasmons in Polycyclic Aromatic Hydrocarbons. ACS Nano 2013, 7, 3635-3643.

24. Lauchner, A.; Schlather, A.; Manjavacas, A.; Cui, Y.; McClain, M. J.; Stec, G. J.; García de Abajo, F. J.; Nordlander, P.; Halas, N. J. Molecular Plasmonics. Nano Lett. 2015, 15, 6208-6214.

25. Dans, P. D.; Walther, J.; Gomez, H.; Orozco, M. Multiscale Simulation of DNA. Curr. Opin. Struct. Biol. 2016, 37, 29-45.

26. Varsano, D.; Di Felice, R.; Marques, M. A. L.; Rubio, A. A TDDFT Study of the Excited States of DNA Bases and Their Assemblies. J. Phys. Chem. B 2006, 110, 7129-7138.

27. M. J. Frisch et al., Gaussian16 Revision A.02, (2016).

28. Chen, J.; Zhang, Y.; Kohler, B. Excited States in DNA Strands Investigated by Ultrafast Laser Spectroscopy. Top Curr. Chem. 2014, 356, 39-87.

29. Wagenknecht, H.; Gray, H. Charge Transfer in DNA: From Mechanism to Application; Wiley: New York, 2005.

30. Chen, C. F.; Park, C. H.; Boudouris, B. W.; Horng, J.; Geng, B.; Girit, C.; Zettl, A.; Crommie, M. F.; Segalman, R. A.; Louie, S. G. et al. Controlling Inelastic Light Scattering Quantum Pathways in Graphene. Nature 2011, 471, 617-620.

31. Silveiro, I.; Plaza Ortega, J. M.; García de Abajo, F. J. Plasmon Wave Function of Graphene Nanoribbons. New J. Phys. 2015, 17, 083013.

32. Yu, R.; Cox, J. D.; Saavedra, J. R. M.; García de Abajo, F. J. Analytical Modeling of Graphene Plasmons. ACS Photon. 2017, 4, 3106-3114.

33. Pines, D.; Nozières, P. The Theory of Quantum Liquids; W. A. Benjamin, Inc.: New York, 1966. 
34. van de Hulst, H. C. Light Scattering by Small Particles; Dover: New York, 1981.

35. Gordon, I. E.; Rothman, L. S.; Hill, C.; Kochanov, R. V.; Tan, Y.; Bernath, P. F.; Birk, M.; Boudon, V.; Campargue, A.; Chance, K. V. et al. The HITRAN2016 Molecular Spectroscopic Database. J. Quant. Spectrosc. Radiat. Transfer 2017, 203, 3-69. 